\title{
ON THE CONCEPT OF ANALYTIC HARDNESS
}

\author{
JANUSZ PAWLIKOWSKI
}

(Communicated by Mirna Dzamonja)

\begin{abstract}
Let $H \subseteq Z \subseteq 2^{\omega}$. Using only classical descriptive set theory we prove that if Borel functions from $2^{\omega}$ to $Z$ give as preimages of $H$ all analytic subsets of $2^{\omega}$, then so do continuous injections. This strengthens a theorem Kechris proved by means of effective descriptive set theory.
\end{abstract}

Let $H \subseteq Z$ be subsets of the Cantor space $\mathcal{C}=2^{\omega}$. The pair $(H, Z)$ is called $\boldsymbol{\Sigma}_{1}^{1}$-hard, resp. Borel $\boldsymbol{\Sigma}_{1}^{1}$-hard, if for any $\boldsymbol{\Sigma}_{1}^{1}$ set $Q \subseteq \mathcal{C}$ there is a continuous, resp. Borel, function $f: \mathcal{C} \rightarrow Z$ with $Q=f^{-1}[H]$. Using effective descriptive set theory Kechris [1] showed that $(H, Z)$ is $\boldsymbol{\Sigma}_{1}^{1}$-hard iff it is Borel $\boldsymbol{\Sigma}_{1}^{1}$-hard. Since the statement of Kechris's theorem is purely classical, one would like to have a classical proof, and, in fact, Kechris asked about a possibility of such a proof.

Using only classical methods we prove the following:

Theorem. Let $n \geq 1$ and $H \subseteq Z \subseteq \mathcal{C}$. If Borel functions from $2^{\omega}$ to $Z$ give as preimages of $H$ all $\boldsymbol{\Sigma}_{n}^{1}(\mathcal{C})$ sets, then so do continuous injections.

Note that for any separable metrizable space $S$ there exists a Borel injection $e: S \rightarrow \mathcal{C}$ whose inverse is continuous (e.g., $e(s)(i)=1 \Leftrightarrow s \in O_{i}$, where $\left\{O_{i}\right\}_{i \in \omega}$ is a basis of $S$ ). Moreover, $e$ can be chosen to be continuous if $S$ is zero-dimensional. It follows that we can change in the Theorem the range space $Z$ to any separable metrizable space, and the domain space $\mathcal{C}$ to any zero-dimensional uncountable Polish space.

Let $X$ be an arbitrary separable metrizable space. The projective classes $\boldsymbol{\Sigma}_{n}^{1}(X)$, $\boldsymbol{\Pi}_{n}^{1}(X)$, and $\boldsymbol{\Delta}_{n}^{1}(X), n \geq 1$, are defined in the same way they are defined for a Polish space (see [2, 25.A]). In particular, $Q \in \boldsymbol{\Delta}_{1}^{1}(X)$ iff $Q \in \boldsymbol{\Sigma}_{1}^{1}(X) \cap \boldsymbol{\Pi}_{1}^{1}(X)$, and if $X$ is a subspace of a Polish space $\bar{X}$, then $Q \in \boldsymbol{\Sigma}_{n}^{1}(X)$ iff there is $\bar{Q} \in \boldsymbol{\Sigma}_{n}^{1}(\bar{X})$ with $Q=X \cap \bar{Q}$.

The $\boldsymbol{\Sigma}_{1}^{1}(X), \boldsymbol{\Pi}_{1}^{1}(X)$, and $\boldsymbol{\Delta}_{1}^{1}(X)$ sets are also called, respectively, analytic, coanalytic, and bianalytic in $X$. Recall that Borel subsets of $X$ are always bianalytic in $X$, and if $X$ is analytic in a Polish space, then the converse is true; there are, however, $X \in \boldsymbol{\Pi}_{1}^{1}(\mathcal{C})$ for which the converse fails.

A function from one separable metrizable space to another is called bianalytic if preimages of open sets are bianalytic (then preimages of bianalytic sets are also bianalytic).

Received by the editors June 4, 2013.

2010 Mathematics Subject Classification. Primary 03E15, 54H05.

The author was supported by grant N N201 418939 of the MNiSW (Ministry of Science and Higher Education). 
Let $\mathcal{P}$ be the set of all nonempty perfect subsets of $\mathcal{C}$ endowed with the Vietoris topology; this is a Polish space, a homeomorph of the Baire space $\omega^{\omega}$. For $G \subseteq \mathcal{C}$, let $\mathcal{P}(G)=\mathcal{P} \cap$ Pow $G$. Recall that if $G$ is $G_{\delta}$ in $\mathcal{C}$, then $\mathcal{P}(G)$ is $G_{\delta}$ in $\mathcal{P}$, and if $G$ is comeager in $\mathcal{C}$, then $\mathcal{P}(G)$ is comeager in $\mathcal{P}$.

For any $Q \subseteq X \times Y, f: X \times Y \rightarrow Z$, and $x \in X$, define the sections $Q_{x} \subseteq Y$ and $f_{x}: Y \rightarrow Z$ by $y \in Q_{x} \Leftrightarrow(x, y) \in Q$ and $f_{x}(y)=f(x, y)$.

Fix also a continuous function $\pi: \mathcal{P} \times \mathcal{C} \rightarrow \mathcal{C}$ such that each section $\pi_{p}, p \in \mathcal{P}$, is a homeomorphism from $\mathcal{C}$ onto $p$ (e.g., let $\pi_{p}$ be induced by the unique bijection from $2^{<\omega}$ onto the split nodes of the tree $\{s \mid l: s \in p, l \in \omega\}$ which preserves the lexicographic ordering).

Proposition. Let $X \subseteq \mathcal{C}$. Given a bianalytic function $b: X \times \mathcal{C} \rightarrow \mathcal{C}$, there exists a bianalytic function $\mathfrak{p}: X \rightarrow \mathcal{P}$ such that for each $x \in X, b_{x} \mid \mathfrak{p}(x)$ is continuous injective or constant.

Proof. Let $B$ consist of all pairs $(x, p) \in X \times \mathcal{P}$ such that $b_{x} \mid p$ is continuous injective or constant.

We claim that (1) $B \in \boldsymbol{\Pi}_{1}^{1}(X \times \mathcal{P})$, and (2) $\forall x \in X B_{x}$ is nonmeager in $\mathcal{P}$; so we can use the "large sections" uniformization for coanalytic sets $([2,36 . F])$ to get a bianalytic $\mathfrak{p}: X \rightarrow \mathcal{P}$ uniformizing $B$.

(1) First, letting $\left\{I_{n}\right\}_{n \in \omega}$ be an enumeration of all clopen subsets of $\mathcal{C}$, note that $b_{x} \mid p$ is continuous iff

$$
\forall n \exists m \forall y \in p \quad y \in I_{m} \quad \Leftrightarrow \quad b(x, y) \in I_{n} .
$$

This defines a $\Pi_{1}^{1}(X \times \mathcal{P})$ set since " $b(x, y) \in I_{n}$ " defines a $\boldsymbol{\Delta}_{1}^{1}(X \times \mathcal{C} \times \omega)$ set.

Next, note that $b_{x} \mid p$ is injective iff

$$
\forall y, y^{\prime} \in p \quad b(x, y)=b\left(x, y^{\prime}\right) \Rightarrow y=y^{\prime},
$$

and constant iff

$$
\forall y, y^{\prime} \in p \quad b(x, y)=b\left(x, y^{\prime}\right) .
$$

Clearly, both these formulas define $\Pi_{1}^{1}(X \times \mathcal{P})$ sets.

(2) Fix $x \in X$. Since the section $b_{x}$ is Borel, it is continuous on a dense $G_{\delta}$ set $G \subseteq \mathcal{C}$. In $G^{2}$ consider the open set

$$
\nabla=\left\{\left(y, y^{\prime}\right) \in G^{2}: b_{x}(y) \neq b_{x}\left(y^{\prime}\right)\right\} .
$$

If the section $b_{x}$ is constant on a nonempty open in $G$ set $U$, then the set $\mathcal{P}_{\text {const }}=\mathcal{P}(U)$ is nonempty and open in $\mathcal{P}(G)$, hence nonmeager in $\mathcal{P}$; clearly $b_{x}$ is constant on each $p \in \mathcal{P}_{\text {const }}$.

Otherwise the set $G^{2} \cap \nabla$ is dense open in $G^{2}$, and then, by the KuratowskiMycielski theorem ([2, 19.1]), the set

$$
\mathcal{P}_{\text {injct }}=\left\{p \in \mathcal{P}: p^{2} \subseteq \nabla \cup\{(y, y): y \in G\}\right\}
$$

is comeager in $\mathcal{P}(G)$, hence also in $\mathcal{P}$; clearly $b_{x}$ is injective on each $p \in \mathcal{P}_{\text {injct }}$.

Corollary. Let $X \subseteq \mathcal{C}$. Given bianalytic functions $b: X \times \mathcal{C} \rightarrow \mathcal{C}$ and $\mathfrak{b}: X \rightarrow \mathcal{P}$, there exists a bianalytic function $\mathfrak{p}: X \rightarrow \mathcal{P}$ such that for each $x \in X, \mathfrak{p}(x) \subseteq \mathfrak{b}(x)$ and $b_{x} \mid \mathfrak{p}(x)$ is continuous injective or constant.

Proof. Get $\mathfrak{p}^{\prime}: X \rightarrow \mathcal{P}$ by the Proposition applied to the function

$$
b^{\prime}(x, y)=b(x, \pi(\mathfrak{b}(x), y)) .
$$


Then the function $x \mapsto \pi_{\mathfrak{b}(x)}\left[\mathfrak{p}^{\prime}(x)\right]$ is our required $\mathfrak{p}$. Just note that the function $\left(p, p^{\prime}\right) \mapsto \pi_{p}\left[p^{\prime}\right]$ is continuous.

Fix now a bianalytic function that is universal for Borel functions. For this, choose $\mathcal{E} \in \boldsymbol{\Pi}_{1}^{1}(\mathcal{C})$ and $U \in \boldsymbol{\Delta}_{1}^{1}(\mathcal{E} \times(\omega \times \mathcal{C}))$ such that $\left\{U_{\varepsilon}\right\}_{\varepsilon \in \mathcal{E}}$ is the family of all Borel subsets of $\omega \times \mathcal{C}$ (see [2,35.B]), and define the function $u: \mathcal{E} \times \mathcal{C} \rightarrow \mathcal{C}$ by

$$
u(\varepsilon, y)(n)=1 \Leftrightarrow(\varepsilon, n, y) \in U .
$$

Then $u$ is bianalytic and $\left\{u_{\varepsilon}\right\}_{\varepsilon \in \mathcal{E}}$ is the family of all Borel functions from $\mathcal{C}$ to $\mathcal{C}$.

Proof of the Theorem. Let $(H, Z)$ be as postulated. For $z \in \mathcal{C}$, define $z^{0} \in \mathcal{C}$ by $z^{0}(i)=z(2 i)$. Let $\mathfrak{p}: X \rightarrow \mathcal{P}$ be obtained by the Corollary applied to $X=\mathcal{E}, b=u$, and $\mathfrak{b}$ given by $\varepsilon \rightarrow\left\{z \in \mathcal{C}: z^{0}=\varepsilon\right\}$.

Consider the following bianalytic injection of $\mathcal{E} \times \mathcal{C}$ into $\mathcal{C}$ :

$$
h(\varepsilon, y)=\pi(\mathfrak{p}(\varepsilon), y) .
$$

If $Q \in \boldsymbol{\Sigma}_{n}^{1}(\mathcal{C})$, then

$$
h[\mathcal{E} \times Q]=\left\{z \in \mathcal{C}: \exists y \in Q h\left(z^{0}, y\right)=z\right\} \in \boldsymbol{\Sigma}_{n}^{1}(h[\mathcal{E} \times \mathcal{C}]) .
$$

Indeed, we have here the projection along $Q \in \boldsymbol{\Sigma}_{n}^{1}(\mathcal{C})$ of the $\boldsymbol{\Delta}_{1}^{1}(h(\mathcal{E} \times \mathcal{C}) \times \mathcal{C})$ set given by the preimage of $\{(z, z): z \in \mathcal{C}\}$ by the bianalytic function

$$
h[\mathcal{E} \times \mathcal{C}] \times \mathcal{C} \ni(z, y) \mapsto\left(h\left(z^{0}, y\right), z\right) .
$$

It follows that $h[\mathcal{E} \times Q]=\tilde{Q} \cap h[\mathcal{E} \times \mathcal{C}]$ for some $\tilde{Q} \in \boldsymbol{\Sigma}_{1}^{1}(\mathcal{C})$. So, by our assumptions about $(H, Z)$, there exists a Borel function $f: \mathcal{C} \rightarrow Z$ such that

$$
h[\mathcal{E} \times Q]=f^{-1}[H] \cap h[\mathcal{E} \times \mathcal{C}],
$$

hence, since $h$ is injective,

$$
\mathcal{E} \times Q=h^{-1}\left[f^{-1}[H]\right]
$$

Find $\varepsilon$ with $f=u_{\varepsilon}$. Then

$$
Q=h_{\varepsilon}^{-1}\left[u_{\varepsilon}^{-1}[H]\right]=\left(u_{\varepsilon} h_{\varepsilon}\right)^{-1}[H] .
$$

The function $u_{\varepsilon} h_{\varepsilon}$ is continuous injective or constant, as $h_{\varepsilon}$ is continuous bijective onto $\mathfrak{p}(\varepsilon)$, and $u_{\varepsilon} \mid \mathfrak{p}(\varepsilon)$ is continuous injective or constant.

If the function $u_{\varepsilon} h_{\varepsilon}$ is injective, we are done. Otherwise it is constant, and it follows that $Q \in\{\mathcal{C}, \varnothing\}$. But then there is a continuous injective $e: \mathcal{C} \rightarrow Z$ with $Q=e^{-1}[H]$, since both sets $H$ and $Z \backslash H$ contain copies of $\left.\mathcal{C} 1\right]$

\section{REFERENCES}

[1] Alexander S. Kechris, On the concept of $\boldsymbol{\Pi}_{1}^{1}$-completeness, Proc. Amer. Math. Soc. 125 (1997), no. 6, 1811-1814, DOI 10.1090/S0002-9939-97-03770-2. MR1372034 (97g:03052)

[2] Alexander S. Kechris, Classical descriptive set theory, Graduate Texts in Mathematics, vol. 156, Springer-Verlag, New York, 1995. MR1321597 (96e:03057)

Department of Mathematics, University of Wroclaw, Pl. Grunwaldzki 2/4, 50-384 Wroclaw, Poland

E-mail address: Janusz.Pawlikowski@math.uni.wroc.pl

1 Fix $G \in G_{\delta}(\mathcal{C}) \backslash F_{\sigma}(\mathcal{C})$. Let $g: \mathcal{C} \rightarrow Z$ be continuous with $G=g^{-1}[H]$. Then $g[G]$ is uncountable, as otherwise $G=g^{-1}[g[G]]$ would be $F_{\sigma}$. Being an uncountable $\boldsymbol{\Sigma}_{1}^{1}$ set, $g[G]$ contains a copy of $\mathcal{C}$. The same argument works for $Z \backslash H$. 